\section{Risk assessment terminology: risk communication part 1}

\author{
Gaetano Liuzzo, ${ }^{1}$ Stefano Bentley, \\ Federica Giacometti, ${ }^{3}$ Silvia Piva, ${ }^{3}$ \\ Andrea Serraino ${ }^{3}$
}

1Modena Local Health Unit, Carpi District, Carpi (MO); ${ }^{2}$ Department of Veterinary

Science, University of Parma, Parma;

${ }^{3}$ Department of Veterinary Medical

Sciences, Alma Mater Studiorum-

University of Bologna, Ozzano dell'Emilia

(BO), Italy

\section{Abstract}

The paper describes the terminology of risk communication in the view of food safety: the theory of stakeholders, the citizens' involvement and the community interest and consultation are reported. Different aspects of risk communication (public communication, scientific uncertainty, trust, care, consensus and crisis communication) are discussed.

\section{English version}

\section{Communication stakeholders}

All those who might have an interest in being part of a risk communication process, or in its results. A list, not necessarily exhaustive might include experts, interest groups local community, risk control agencies, health authorities (both public and private) educational system, media (Biocca, 2002).

\section{Stakeholders (theory)}

According to this theory, from the economic point of view, stakeholders are all individuals and groups who may have an interest (stake), i.e. something to gain or lose or something at risk in the company's activity (Gilardoni, 2000).

\section{Citizens' involvement}

A process of informed dialogue and deliberation among citizens, which usually involves the exchange of ideas and values, the possibility of mutual learning, decision making on the best ways to proceed, as well as the starting of partnerships for creating solutions (Santé Canada, 2000).

\section{Risk communication}

Legal definition: risk communication means the interactive exchange of information and opinions throughout the risk analysis process as regards hazards and risks, risk-related factors and risk perceptions, among risk asses- sors, risk managers, consumers, feed and food businesses, the academic community and other interested parties, including the explanation of risk assessment findings and the basis of risk management decisions (European Comission, 2002).

The interactive exchange of information and opinions as regards risks and risk management among risk assessors, risk managers, consumers and other interested parties (CAC, 2004). The interactive exchange of information and opinions throughout the risk analysis process as regards hazards and risks, riskrelated factors and risk perceptions, among risk assessors, risk managers, consumers, feed and food businesses, the academic community and other interested parties, including the explanation of risk assessment findings and the basis of risk management decisions (European Comission, 2002).

\section{Public communication on risk}

Risk communication coming from a public institution, in line with Lasswell's postulate. It is thus made by a public administration, which advertises its activity, task and identity by means of any available media, addressing citizens and organisations (external communication) as well as other members of institution (internal communication). Thereby, it guarantees citizens' right of information and promotes active and substantial expression of citizenship rights (Grandi, 2007).

\section{Communication and scientific uncertainty}

Four different certainty/uncertainty scenarios have been described (OMF/FAO/OIE, 2001), which justify different risk communication messages: i) issues where we think we know the answers recognising that new data can change the conclusions (high certainty); ii) issues where we do not know (high uncertainty); iii) issues where there is debate or controversy (moderate uncertainty); iv) new emerging issues of potential risk.

\section{Risk communication (types)}

Lundgren and McMakin (2013) distinguish between: care communication, consensus communication and crisis communication. In care communication, the risk and the way to manage it are often well known; the goal is to promote a change in individual behaviour, as well as the context which influences personal choices (e.g., unhealthy food habits). In consensus communication, scientific knowledge can be more controversial; the goal of this type of risk communication is to inform and encourage groups to work together to reach a shared decision about how the risk will be managed (e.g., food, OGM). Crisis communication has the objective of directing people's fear towards sort of informed alert status so that they would adopt health protection and person-
Correspondence: Gaetano Liuzzo, Modena Local Health Unit, Carpi District, Piazzale Donatori di Sangue 3, 41012 Carpi (M0), Italy.

Tel. +39.059.659949 - Fax: +39.059 .659994 .

E-mail: g.liuzzo@ausl.mo.it

Key words: Risk communication; Consultation; Participation; Trust.

Received for publication: 26 November 2015.

Accepted for publication: 29 November 2015.

This work is licensed under a Creative Commons Attribution-NonCommercial 4.0 International License (CC BY-NC 4.0).

(C) Copyright G. Liuzzo et al., 2016

Licensee PAGEPress, Italy

Italian Journal of Food Safety 2016; 5:5648

doi:10.4081/ijfs.2016.5648

al safety behaviors in case of emergency (e.g. an outbreak).

\section{Community of interest}

Group of participants, gathering the involved in a matter, a view or a common concern, usually for a limited period (Santé Canada, 2000).

\section{Consultation}

The solicitation to express points of view from participants in the process of developing public health policies, often through forums, round tables or consultation councils. Consultation is a part of the participation process, but not its synonym (Santé Canada, 2000).

\section{Elements of communication}

The elements of communication are: the issuer, the contact, the message, the code, the context and the recipient. The issuer (individual, group or institution) sends a message to a recipient (individual, group or institution). For the message to reach the recipient, a contact and a channel are necessary (the Worldwide Web, the simple air for a voice message). The message can be understood only if issuer and recipient share the same code, e.g.set of rules that determines how to give a meaning to the signs that build up the message. The context is what comes along with the message, first of all the circumstances in which this is expressed (Grandi, 2007).

\section{Trust}

The belief that the majority of future events will realize as expected (Siegrist et al., 2003). The most accredited theories suggest that trust is substantially based on three factors: the perception that the other party is benevolent (shall be doing things for my own good), the perception that the other party is competent (shall be choosing the best option), and 
the perception that we both have similar values (e.g., stealing is bad, killing is a sin). When a food scandal breaks out, showing own innocence with facts and figures proves pointless, unless a solid ground of shared values has been previously built. And if this has not been done, promoting a communication which emphasises that the company and the people have the same values is mandatory (Salvadori and Rumiati, 2005).

\section{Social trust}

The willingness to trust those who have responsibility in decision-making and actions related to the management of technology, environment, medicine or other areas of public health and safety (Siegrist and Cvetkovich, 2000).

\section{Participant}

A person, group or organisation that has interests at stake in its solutions and in the overall public participation process (Santé Canada, 2000).

\section{Participation}

A series of activities and relationships that include: two-way communication, public education, consultation of the participants, the involvement of citizens, advisory bodies, partnerships, joint decision-making power. It includes continuous exchanges between participants who agree to mutually influence on decisions to be taken (Santé Canada, 2000).

\section{Italian version}

\section{Attori dei processi comunicativi (stakeholders)}

Tutti coloro che hanno un interesse nella partecipazione o nei risultati di un processo di comunicazione sul rischio. Un elenco, non necessariamente esaustivo, comprende: le persone esperte, le autorità amministrative, i gruppi d'interesse, la comunità locale, le agenzie responsabili della vigilanza sul rischio, le organizzazioni sanitarie e di assistenza pubbliche e private, il sistema educativo, gli organi d'informazione, gli esperti del settore (Biocca, 2002).

\section{Stakeholders (teoria degli)}

Secondo questa teoria, dal punto di vista economico, sono stakeholder tutti i soggetti e i gruppi che possono avere un interesse (stake) ossia qualcosa da guadagnare 0 da perdere, qualcosa a rischio nell'attività dell'impresa (Gilardoni, 2000).

\section{Coinvolgimento dei cittadini}

Processo di dialogo informato e di deliberazione fra i cittadini, comprende abitualmente lo scambio d'idee e di valori, la possibilità d'apprendere gli uni dagli altri, le prese di decisione sui migliori modi di procedere, così come la creazione di paternariati per dare vita a delle soluzioni (Santé Canada, 2000).

\section{Comunicazione sul rischio}

Definizione legale: comunicazione del rischio, lo scambio interattivo, nell'intero arco del processo di analisi del rischio, d'informazioni e pareri riguardanti gli elementi di pericolo e i rischi, i fattori connessi al rischio e la percezione del rischio, tra responsabili della valutazione del rischio, responsabili della gestione del rischio, consumatori, imprese alimentari e del settore dei mangimi, la comunità accademica e altri interessati, ivi compresi la spiegazione delle scoperte relative alla valutazione del rischio e il fondamento delle decisioni in tema di gestione del rischio (Commissione Europea, 2002). Scambio interattivo d'informazioni e di opinioni sui rischi e la gestione dei rischi fra i responsabili della loro valutazione e della loro gestione, i consumatori e le altre parti interessate (CAC, 2004). Lo scambio interattivo, nell'intero arco del processo di analisi del rischio, d'informazioni e pareri riguardanti gli elementi di pericolo e i rischi, i fattori connessi al rischio e la percezione del rischio, tra responsabili della valutazione del rischio, responsabili della gestione del rischio, consumatori, imprese alimentari e del settore dei mangimi, la comunità accademica e gli altri interessati, ivi compresi la spiegazione delle scoperte relative alla valutazione del rischio e il fondamento delle decisioni in tema di gestione del rischio (Commissione Europea, 2002).

\section{Comunicazione pubblica sul rischio}

La comunicazione sul rischio dell'istituzione pubblica che rispetta il postulato di Lasswell. È quella comunicazione cioè realizzata da una pubblica amministrazione che pubblicizza la propria attività, funzione e identità, attraverso tutti i media a sua disposizione, rivolgendosi ai cittadini o alle organizzazioni (comunicazione esterna) e a chi opera all'interno delle istituzioni (comunicazione interna). Garantisce in questo modo il diritto dei cittadini all'informazione; promuove l'identità dell'ente pubblico; offre la possibilità ai cittadini di esprimere in maniera attiva e sostanziale i diritti di cittadinanza (Grandi, 2007).

\section{Comunicazione e incertezza scien- tifica}

Sono quattro le situazioni differenti di certezza/incertezza scientifica che sono state descritte (OMS/FAO/OIE, 2001) e che giustificano messaggi distinti in materia di comunicazione: i) problemi per i quali si pensa di conoscere le risposte ma si riconosce altresì l'eventualità che nuovi dati possono modificare le conclusio- ni: certezza elevata; ii) problemi per i quali si riconosce ignoranza: incertezza elevata; iii) problemi che danno luogo ad un dibattito e controversie: incertezza moderata; i) problemi nuovi sostenuti da pericoli potenziali.

\section{Comunicazione sui rischi (tipi)}

Lundgren e McMakin (2013) distinguono: care communication, consensus communication e crisis communication. Nel caso della care communication il rischio è spesso ben conosciuto e si sa come affrontarlo, l'obiettivo è favorire il cambiamento comportamentale sull'individuo e sul contesto che influenza le sue scelte (es. alimentazione scorretta). La consensus communication è quella comunicazione in cui le conoscenze scientifiche sul rischio sono più controverse, obiettivo di questo tipo di comunicazione è quello di informare e incoraggiare i diversi attori sociali a lavorare insieme per ricercare una decisione condivisa su come gestire il rischio (es. alimenti OGM). La crisis communication ha l'obiettivo di incanalare la paura della gente in uno stato di preoccupazione informata e vigile che aiuti le persone ad adottare comportamenti di protezione della salute e sicurezza personale in caso di emergenza (es. comparsa di un'epidemia).

\section{Comunità d'interesse}

Gruppo di partecipanti che riunisce gli interessati a una questione, di un'opinione o di una preoccupazione comune, spesso per un periodo di tempo limitato (Santé Canada, 2000).

\section{Consultazione}

La sollecitazione a esprimere punti di vista da parte dei partecipanti nel contesto del processo di elaborazione di politiche di sanità pubblica, spesso attraverso la realizzazione di forum, tavole rotonde 0 consigli consultativi. La consultazione è parte del processo di partecipazione ma non né è sinonimo (Santé Canada, 2000).

\section{Elementi della comunicazione}

Sono elementi della comunicazione: l'emittente, il contatto, il messaggio, il codice, il contesto, il destinatario. L'emittente (persona, gruppo o istituzione) invia un messaggio a un destinatario (persona, gruppo o istituzione). Affinché il messaggio raggiunga un destinatario è necessario che tra i due vi sia un contatto e quindi un canale (es. rete Internet o l'atmosfera nel caso di messaggio verbale). Il messaggio può essere compreso solo se i due condividono un codice. Il codice è un sistema di regole che stabilisce come si trasformano i segni di cui è composto il messaggio in significati. Il contesto è ciò di cui si comunica, ciò che accompagna il messaggio, in primis le circostanze nelle quali questo è espresso (Grandi, 2007). 


\section{Fiducia}

È la convinzione che la maggior parte degli eventi futuri si realizzi come atteso (Siegrist, et al., 2003). Secondo le teorie più accreditate tre sono i fattori che stanno alla base della fiducia: la percezione che l'altra parte sia benevola (ovvero faccia le cose per il mio bene), la percezione che l'altra parte sia competente (ovvero, faccia le cose migliori), la percezione che io e l'altra parte abbiamo dei valori simili (es. rubare è male, uccidere è peccato). Quando esplode uno scandalo alimentare, non conta tanto dimostrare con dati e fatti alla mano la propria innocenza, quanto piuttosto l'avere costruito prima un terreno di valori condivisi. E se non lo si è fatto prima, è necessario e importante promuovere una comunicazione che enfatizzi come l'azienda e le persone abbiano gli stessi valori (Salvadori e Rumiati, 2003).

\section{Fiducia sociale}

La disponibilità a fidarsi di chi ha responsabilità nella presa di decisioni e azioni relative alla gestione della tecnologia, dell'ambiente, della medicina 0 altri settori della salute pubblica e della sicurezza (Siegrist e Cvetkovich, 2000).

\section{Partecipante}

Una persona, un gruppo od un organismo che ha interessi in gioco, nelle sue soluzioni e nel processo generale di partecipazione del pubblico (Santé Canada, 2000).

\section{Partecipazione}

Una serie di attività e di relazioni che comprendono: la comunicazione nei due sensi; l'educazione del pubblico; la consultazione degli intervenuti; il coinvolgimento dei cittadini, gli organismi consultivi, i paternariati, il potere di decisione congiunto. Comprende gli scambi continui fra partecipanti che accettano di influenzarsi mutuamente sulle decisioni da prendere (Santé Canada, 2000).

\section{References}

Biocca M, 2002. La comunicazione sul rischio per la salute nel teatro di Sagredo. Centro Scientifico Editore, Turin, Italy.

CAC, 2004. Textes de base concernant l'hygiene alimentaire. Codex Alimentarius Commission, Rome, Italy.

European Commission, 2002. Regulation of the European Parliament and of the Council of 28 January 2002 laying down the general principles and requirements of food law, establishing the European Food Safety Authority and laying down procedures in matters of food safety, 178/2002/CE. In: Official Journal, L 31/1,
01/02/2002.

Gilardoni A, 2000. Health, safety and environment. Indirizzi strategici e problematiche operative. Egea ed., Milan, Italy.

Grandi R, 2007. La comunicazione pubblica. Carocci ed., Rome, Italy.

Lundgren R, McMakin A, 2013. Risk communication: a handbook for communicating enviromental safety and health risks. Wiley-IEEE Press, Hoboken, NJ, USA.

OMS/FAO/OIE, 2001. Consultation technique conjointe OMS/FAO/OIE sur l'ESB: santé publique, santé animale et commerce, Siège de l'0.I.E, Paris, June 11-14 2001. Conclusions et principales recommandations. Available from: www.oie. int/doc/ged/D6713.PDF

Salvadori L, Rumiati R, 2005. Nuovi rischi, vecchie paure. Il Mulino ed., Bologna, Italy.

Santé Canada, 2000. Participation du public: cadre et lignes directrices. Bureau de la participation des consommateurs et du public Santé Canada, Ministre de Travaux publics et Services gouvernementaux du Canada, Ottawa, Canada.

Siegrist M, Cvetkovich G, 2000. Perception of hazards: the role of social trust and knowledge. Risk Anal 20:713-9.

Siegrist M, Earle TC, Gutscher H, 2003. Test of a trust and confidence model in the applied context of electromagnetic field (EMF). Risk Anal 23:705-16. 